\title{
Analysis of Static and Dynamic Load on Hydrostatic Bearing with Variable Viscosity and Pressure
}

\author{
V. Srinivasan* \\ Professor, School of Mechanical Engineering, Bharath University, Chennai-73; srinivasan_v9669@yahoo.co.in
}

\begin{abstract}
Hydrostatic bearing finds wide application in machine tools with various technologies because of their high stiffness and damping characteristic. The environmental conditions, such as low and/or high temperatures, dust and dirt, moisture and unusual mounting conditions, can also affect a bearing's performance adversely. Therefore, both mechanical and environmental factors may affect the choice of a bearing and its performance. For high speed applications it is necessary to have design data including the effect of rotational lubricant inertia.The objective of the study is to design a hydrostatic bearing with following properties such as high stiffness, damping characteristic and lubrication inertia. In the present study Reynolds equation is used and boundary conditions are changed for various parameters such as temperature distribution, viscosity variation and radial load. The simulated results were analyzed in detail and found that increasing the viscosity of hydrostatic thrust bearing under specific conditions when both surfaces are rotated, the wear and tear are minimized and life time has been increased. This will be of great use in high speed applications.
\end{abstract}

Keywords: Hydrostatic Bearing, Viscosity, Pressure, Temperature, Reynolds Equation, Lubricant.

\section{Introduction}

It is customary in the theory of lubrication to assume that the influence of the inertia terms in the equations of motion are negligible compared with the effect of the viscous term. When the lubricant gap and the flow rate are small, the pressure falls logarithmically in a radial direction towards the edge of the bearing, as indicated by the exact solution of the Navier Stokes equations. Studies ignoring the inertia terms and assuming negligible transverse velocity components has been reported [1].

When the lubricant gap and flow rate are large, the viscosity variation is governed mainly by the inertia terms in the equations of motion and positive pressure gradients are encountered at the smaller radii and negative pressure gradients further out towards the edge of the bearing. Solutions for the condition of large lubricant gap and flow rate for stationary parallel discs are well known. The radial inertia of the lubricant can create a significant change in the viscosity variation, particularly when air is used as the lubricant, resulting in a loss in the load capacity of the bearing [2].

In the practical applications of externally pressurized thrust bearings, it is usual for one of the bearing surfaces to rotate. As the speed of rotation increases, the centripetal inertia term become dominant relative to the viscous term reducing the overall viscosity variation. Researchers have investigated this particular case, deriving expressions for the viscosity variation for plane and stepped bearings $[3,4]$. The same was revealed by other wonders that the importance of both the radial and the rotational inertia terms in the equations of motion has recently increased due to extreme bearing operating conditions [5].

Hydrostatic thrust bearings characteristics: High loadcarrying capacity, zero wear of bearing surfaces, low friction

*Corresponding author:

V. Srinivasan (srinivasan_v9669@yahoo.co.in) 


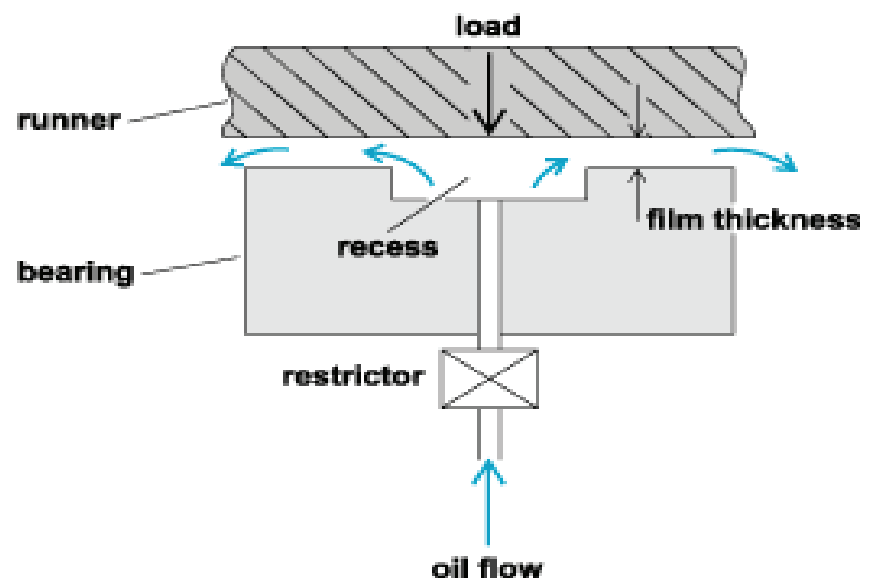

Figure 1. Pad thickness (load and bearing).

at low or zero speeds, large fluid film stiffness and damping, as shown in Figure 1 reduced vibrations and good positional accuracy [6]. Different bearing configurations were considered and it was reported that the addition of the stem enabled this bearing to support radial loads [2]. Analytically studied the performance of circular thrust pad hydrostatic bearing of various recess shapes, i.e., triangular, square, rectangular etc. They compared results with the solutions obtained by an electrical analog technique.

Owing to rapid technological advancements in manufacturing techniques, super heavy constant flow hydrostatic thrust bearing having sector recess can be used widely in the many industrial heavy applications.

Inorder toprovidereasonable data for design, lubrication, thermal deformation and force deformation computation for hydrostatic thrust bearing in the heavy equipment, it is important to compute viscosity variation, temperature distribution and oil flow rate of the super heavy constant flow hydrostatic thrust bearing having sector recess [7].

Flat-land thrust bearings are the simplest and least expensive to make. They handle light loads for simple positioning of rotors in electric motors, appliances, crankshafts, and other machinery. Flat-land bearings carry 10 to $20 \%$ the load of other thrust-bearing types. Previous studies reveal that flat parallel surfaces do not directly build oil-film pressure through pumping action [8] as shown in Figure 2. They depend instead on thermal expansion of both the oil film and bearing surface to generate an oil-supporting wedge.

Self-acting hydro dynamically lubricated slider bearings have, however, certain important disadvantages:

1. If the design speed is low, it may not be possible to generate sufficient hydrodynamic pressure.

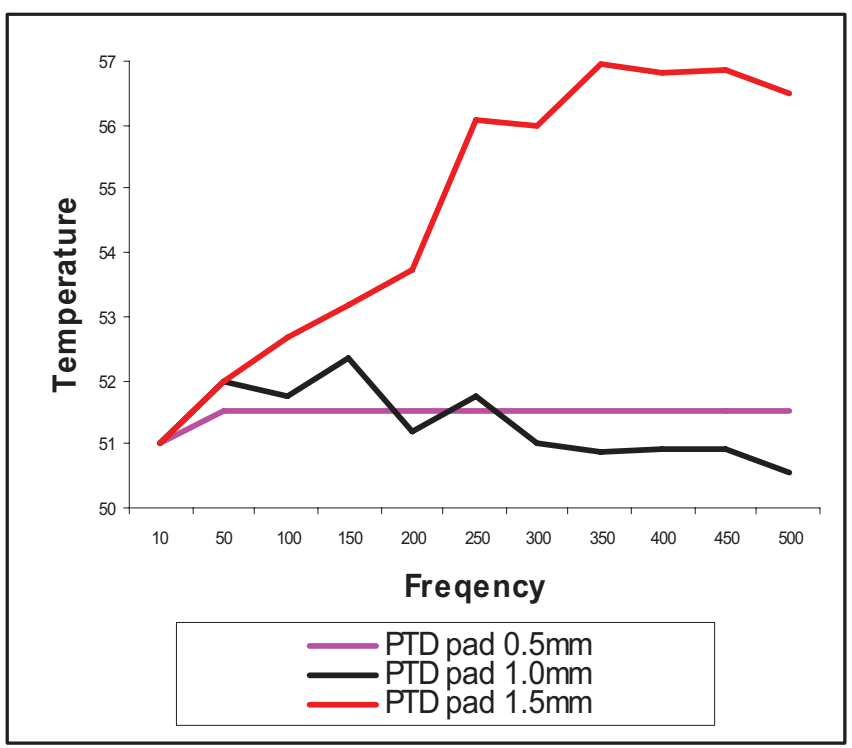

Figure 2. Frequency with thickness of pad.

2. Fluid film lubrication may break down during starting, direction changing, and stopping.

3. In a journal bearing the shaft runs eccentrically and the bearing location varies with load, thus implying low stiffness.

In hydrostatic also called "externally pressurized" lubricated bearings the bearing surfaces are separated by a fluid film maintained by a pressure source outside the bearing [9]. Hydrostatic bearings avoid disadvantages 1 and 2 and reduce the variation of bearing location with load mentioned in disadvantage 3 . The characteristics of hydrostatically lubricated bearings are:

1. Extremely low friction

2. Extremely high load-carrying capacity at low speeds

3. High positional accuracy in high-speed, light-load applications

4. A lubrication system more complicated than that for self-acting bearings. Therefore, hydrostatically lubricated bearings are used when the requirements are extreme as in large telescopes and radar tracking units, where extremely heavy loads and extremely low speeds are used, or in machine tools and gyroscopes, where extremely high speeds, light loads, and gas lubricants are used.

Fluid bearings use a thin layer of liquid or gas fluid between the bearing faces, typically sealed around or under 
the rotating shaft. There are two principal ways of getting the fluid into the bearing:

- In fluid static, hydrostatic and many gas or air bearings, the fluid is pumped in through an orifice or through a porous material.

- In fluid-dynamic bearings, the bearing rotation sucks the fluid on to the inner surface of the bearing, forming a lubricating wedge under or around the shaft.

Hydrodynamic bearings rely on bearing motion to suck fluid into the bearing and may have high friction and short life at speeds lower than design or during starts and stops. An external pump or secondary bearing may be used for startup and shutdown to prevent damage to the hydrodynamic bearing [10].

A secondary bearing may have high friction and short operating life, but good overall service life if bearing starts and stops are infrequent.

The thin films can be thought to have pressure and viscous forces acting on them. Because there is a difference in velocity there will be a difference in the surface traction vectors [11]. Because of mass conservation we can also assume an increase in pressure, making the body forces different.

Fluid bearings can be relatively cheap compared to other bearings with a similar load rating. The bearing can be as simple as two smooth surfaces with seals to keep in the working fluid [12].

In contrast, a conventional rolling-element bearing may require many high-precision rollers with complicated shapes. Hydrostatic and many gas bearings do have the complication and expense of external pumps.

\section{Objectives}

To analyse the viscosity variation of the lubricant used for the hydrostatic thrust bearing.

To analyse the load carrying capacity of the lubricant used for the hydrostatic thrust bearing.

To analyse the static and dynamic characteristic of various film thickness in hydrostatic thrust bearing.

To analyse the temperature variation of the lubricant used for the hydrostatic thrust bearing.

\section{Research Analysis Modeling}

The research deals with the designing of a complex hydrostatic bearing of self-acting type satisfying the requiring design. The pressure pads in hydrostatic bearings carry the load capacity.

Most hydrostatic systems (thrust or journal bearings) use several evenly spaced pads so nonsymmetrical load distributions can be handled. To estimate performance, pads can be treated separately.

Pressure is maintained in the recess by fluid pumped through a flow restrictor. The pressure lifts the rotor until flow out of the recess and over the land equals flow in.

A constant gap is maintained for a given recess pressure and bearing load. The gap establishes the volume of fluid pumped through the bearing. An alternative design is to connect a fluid displacement pump (gear or vane type) directly to each pocket without flow restriction.

Pressurizing pump power can be estimated from the product of pad pressure and total flow through the pads. For an estimate of lubricant flow rate, a gap (or film thickness) is assumed -- typical values are 0.001 to $0.002 \mathrm{in}$.

Design of flow restrictors influences bearing stiffness, pumping power, supply pressure, and lubricant flow. A flow restrictor is necessary to provide a pressure drop between the supply manifold and the pad recesses to ensure pressure requirements in any given pad never exceed supply pressure. A bearing with restrictors is called a "compensated bearing."

Action of the restrictors is such that if the thrust load is centered, pressure in all the pads is equal and lower than pump pressure. If the load is off center, the gap decreases somewhat on the loaded side and flow from the pad also decreases. This results in an increase in loaded-pad pressure and a decrease in pad pressure on the opposite side. The runner automatically seeks a nearly level attitude.

The boundary conditions for slip flow at the surface of a gas bearing can be written as.

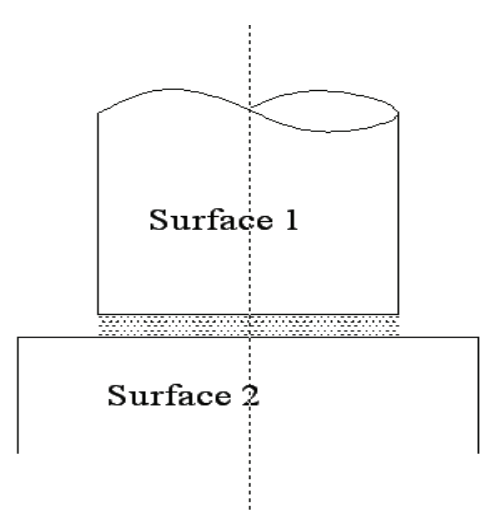

Figure 3. Bearing surface contact. 


$$
u_{\text {slip }}=\sigma\left(\frac{2-f}{f}\right) \lambda\left(\frac{\partial u}{\partial z}\right)_{\text {wall }}
$$

where $f$ is the reflection coefficient, $\lambda$ is the mean free path and $\sigma$ is a numerical constant. Because $\sigma$ and $f$ are close to unity it can be assumed that $\sigma(2-f) / f$ is unity. As the molecular mean free path $\lambda$ depends upon fluid viscosity, pressure and temperature it can be approximated by the relation.

$$
\lambda=\frac{16}{5(2 \pi)^{1 / 2}} \frac{\eta}{p}(R T)^{1 / 2}
$$

where $R$ is the gas constant, $T$ the temperature of the gas, $\eta$ the viscosity of the gas and $P$ its pressure.

The effect of slip is also important on the flow behaviour of liquids especially when the bearing surface is very smooth and is operating at higher surface temperatures as shown in Figure 3 where the viscosity of the base oil decreases near the surface. This effect has been studied for liquids. The slip velocity at the will can be written as

$$
u_{\text {slip }}=\frac{1}{\beta}\left(\eta \frac{\partial u}{\partial z}\right)_{\text {wall }}
$$

where $\beta$ is the coefficient of sliding friction at the wall and $\eta$ is the liquid viscosity.

\subsection{Reynolds Equation}

The physical configuration of fluid flow between two curved surfaces is shown in Figure 1.

The basic equations of motion and the equation of continuity for a Newtonian fluid considering the variation of fluid properties both across and along the film thickness can be written as

$$
\begin{aligned}
& \rho \frac{D u}{D t}=\rho X-\frac{\partial p}{\partial x}+\frac{2}{3} \frac{\partial}{\partial x}\left\{\eta\left(\frac{\partial u}{\partial x}-\frac{\partial v}{\partial y}\right)\right\}+ \\
& \frac{2}{3} \frac{\partial}{\partial x}\left\{\eta\left(\frac{\partial u}{\partial x}-\frac{\partial w}{\partial z}\right)\right\}+ \\
& +\frac{\partial}{\partial y}\left\{\eta\left(\frac{\partial v}{\partial x}+\frac{\partial u}{\partial y}\right)\right\}+\frac{\partial}{\partial z}\left\{\eta\left(\frac{\partial u}{\partial z}+\frac{\partial w}{\partial x}\right)\right\} \\
& \rho \frac{D v}{D t}=\rho Y-\frac{\partial p}{\partial y}+\frac{2}{3} \frac{\partial}{\partial y}\left\{\eta\left(\frac{\partial v}{\partial y}-\frac{\partial u}{\partial x}\right)\right\}+ \\
& \frac{2}{3} \frac{\partial}{\partial y}\left\{\eta\left(\frac{\partial v}{\partial y}-\frac{\partial w}{\partial z}\right)\right\}+
\end{aligned}
$$

$$
\begin{aligned}
& +\frac{\partial}{\partial z}\left\{\eta\left(\frac{\partial w}{\partial y}+\frac{\partial v}{\partial z}\right)\right\}+\frac{\partial}{\partial x}\left\{\eta\left(\frac{\partial v}{\partial x}+\frac{\partial u}{\partial y}\right)\right\} \\
& \rho \frac{D w}{D t}=\rho Z-\frac{\partial p}{\partial z}+\frac{2}{3} \frac{\partial}{\partial z}\left\{\eta\left(\frac{\partial w}{\partial z}-\frac{\partial u}{\partial x}\right)\right\}+ \\
& \frac{2}{3} \frac{\partial}{\partial z}\left\{\eta\left(\frac{\partial w}{\partial z}-\frac{\partial v}{\partial y}\right)\right\}+ \\
& +\frac{\partial}{\partial x}\left\{\eta\left(\frac{\partial u}{\partial z}+\frac{\partial w}{\partial x}\right)\right\}+\frac{\partial}{\partial y}\left\{\eta\left(\frac{\partial w}{\partial y}+\frac{\partial v}{\partial z}\right)\right\} \\
& \frac{\partial}{\partial t}+\frac{\partial}{\partial x}(\rho u)+\frac{\partial}{\partial y}(\rho v)+\frac{\partial}{\partial z}(\rho w)=0
\end{aligned}
$$

With the usual assumptions of lubrication theory equations. can be simplified to

$$
\begin{aligned}
& \frac{\partial p}{\partial x}=\frac{\partial}{\partial z}\left(\eta \frac{\partial u}{\partial z}\right) \\
& \frac{\partial p}{\partial y}=\frac{\partial}{\partial z}\left(\eta \frac{\partial v}{\partial z}\right)
\end{aligned}
$$

where $p=p(x, y)$ is the film pressure.

Equation represents a generalized form of Reynolds equation for compressible fluid film lubrication considering slip velocities at the bearing surfaces.

The two sets of functions $F$ and $G$ depend upon the variation of fluid properties both along and across the film and on the slip conditions at the surfaces which is shown in Figure 4.

The viscosity of the lubricant can vary across the film thickness which is shown in Figure 5 and may be different near the bearing surfaces owing to the reaction of additives

\section{Temperature due to Oil Film Pressure}

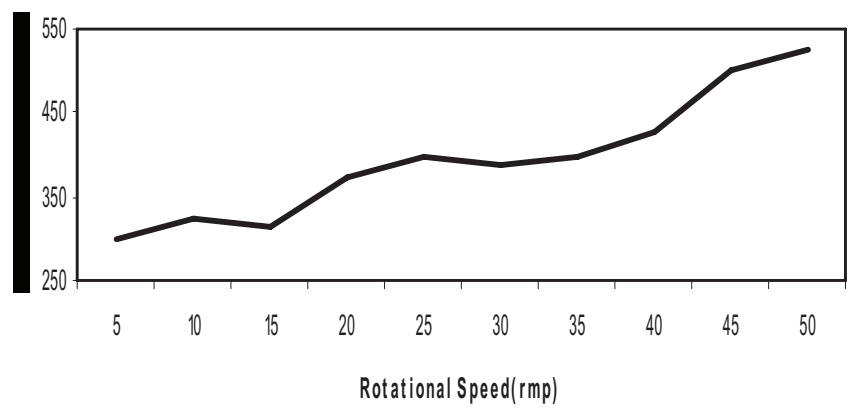

Figure 4. Temperature distribution curve. 


\section{Viscosity variation across the film}

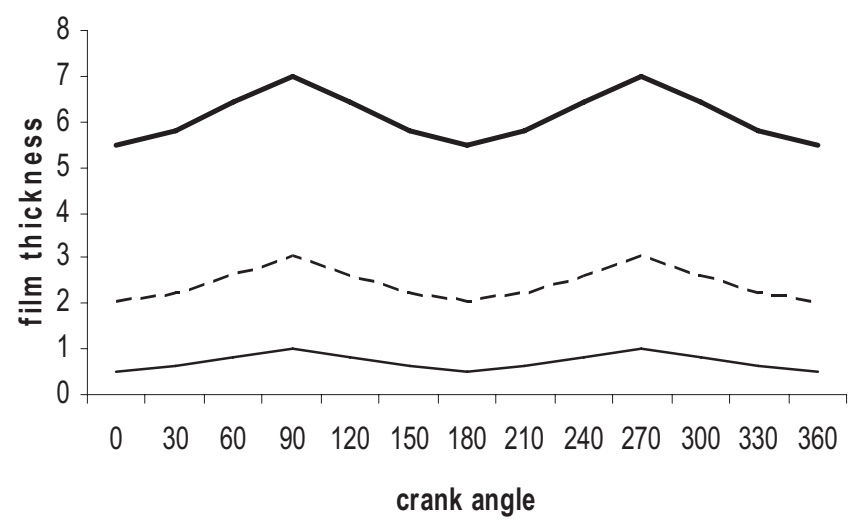

Figure 5. Temperature distribution curve with various viscosities.

and surfactants with the surfaces. The most general form of Reynolds equation to study such a situation is given by the equation

$$
\frac{\partial}{\partial t}+\frac{\partial}{\partial x}(\rho u)+\frac{\partial}{\partial y}(\rho v)+\frac{\partial}{\partial z}(\rho w)=0
$$

Considering a reasonable case where the density and viscosity of the lubricant near the bearing surfaces may be different from that of the central region gives the viscosity changes.

The pressure boundary condition necessary for the solution of eqn.(8) in general are not satisfactorily known and hence a one dimensional analysis is carried out in Reynold equation 7(a) to study qualitatively the effect of pseudo plasticity on piston ring lubrication.

In order to increase rotational speed and bearing capacity of a constant flow hydrostatic thrust bearing, a theoretical study concerning lubrication performance of a super heavy constant flow hydrostatic thrust bearing having sector recess is described.

The Computational Fluid Dynamics and the Finite Volume Method have been used to compute the lubrication characteristics of a super heavy constant flow hydrostatic thrust bearing, such as recess pressure, recess temperature and oil flow rate.

This study theoretically analyzes the influence of workbench rotational speed on the bearing lubrication performance according to computational fluid dynamics and lubricating theory.

It has revealed its viscosity variation law, temperature distribution law and oil flow rate.
Liquid hydrostatic thrust bearing working principle is that lubricating oil which is compulsively injected into oil cavity forms bearing capacity of hydrostatic bearing through throttling action of the gap between resistive oil edges and the rotary table, lifts bearing spindle, and bears external loads.

The working principle of hydrostatic bearing with quantitative oil supply is shown as Figure 1. Lubricating oil enters into oil cavity from pump along inlet and flows out along the radial shallow recess and resistive oil edges of external ring as shown in Figure 1.

Flow mobility of the fluid between HIP round rail and rotating worktable must meet mass conservation, momentum conservation and energy conservation.

\subsection{Mass Conservation Equation}

The law of mass conservation is the basic law which meets any mobile system. Mass conservation equation is:

$$
\frac{\partial \rho}{\partial t}+\frac{\partial(\rho u)}{\partial x}+\frac{\partial(\rho v)}{\partial y}+\frac{\partial(\rho w)}{\partial z}=0
$$

where $\rho$ is density $(\mathrm{kg} / \mathrm{m} 3) ; t$ is time $(\mathrm{s}) ; u, v$ and $w$ are in the $x, y$ and $z$ direction component of speed vector $\bar{v}$.

Flow capacity is $0.098 \mathrm{~kg} / \mathrm{s}$, external pressure is $0.1 \mathrm{Mpa}$, the worktable rotational speed of $2.5 \mathrm{rpm}, 8 \mathrm{rpm}, 10 \mathrm{rpm}$, $12.5 \mathrm{rpm}, 16 \mathrm{rpm}, 20 \mathrm{rpm}, 25 \mathrm{rpm}, 31.5 \mathrm{rpm}$ and $40 \mathrm{rpm}$, the three-dimensional pressure fields, temperature fields and flow fields. The recess and obviously overlapping in the oil cavities, the flow state is from laminar flow to turbulent flow.

\section{Findings}

This research after using the modified Reynolds equations assuming that there is a relation between the viscosity and film thickness determines the flow, the load, and the pumping power loss for bearing. The simulation model has established that there is a relationship between hydrostatic thrust bearing performance and various film thickness in recess pad. The simulation model has also proved that there is decrease in the viscosity variation due to increasing the viscosity of hydrostatic thrust bearing under the condition when one bearing surface is rotated. The simulation model nothing but Reynold equation

$$
\frac{\partial}{\partial t}+\frac{\partial}{\partial x}(\rho u)+\frac{\partial}{\partial y}(\rho v)+\frac{\partial}{\partial z}(\rho w)=0
$$


has also proved that there is decrease in the viscosity variation or temperature due to increasing the viscosity of hydrostatic thrust bearing under the condition when both bearing surface is rotated.

The hydrostatic thrust bearings to reduce the numbers of parts and size, and to eliminate expensive mineral lubricant storage and pumping, thus further satisfying stringent environmental constraints. Despite the many advantages offered by hydrostatic bearings, rotor dynamic instabilities due to hydrodynamic (shear flow) and fluid compressibility effects are issues of primary concern for high speed operation with large pressure differentials.

Laboratory measurements of load, leakage, orque, and identification of rotor dynamic force coefficients aided to benchmark are compared with simulation model technique. The agreement also been tested.

\section{Conclusion}

The lubrication characteristics of a super heavy constant flow hydrostatic thrust bearing, such as recess pressure, recess temperature and oil flow state are computed according to the Computational Fluid Dynamics and the Finite Volume Method. This study theoretically analyzes the influence of workbench rotating velocity on the viscosity variation law, temperature distribution law and oil flow state.

The following conclusions are made based on the numerical calculations done by iterative method for the two-dimensional oil film pressure field state of a super heavy constant flow hydrostatic thrust bearing by the usage of the fluid dynamics, lubricating theory and the Finite Volume Method.

The results show that oil cavity pressure is almost invariant by increasing of workbench rotating velocity, oil cavity temperature is increasing gradually by increasing of workbench rotating velocity. Each type of bearing has unique geometric and manufactured features that influence the amount of friction that occurs during operation. This, combined with factors discussed previously about how an application can influence heat generation, underscores the fact that a power transmission system is a complex assembly that interacts with its surrounding environment.

\section{References}

1. Chattopadhyay A K, Majumdar B C (1984). Steady state solution of finite hydrostatic porous oil journal bearing with tangential velocity slip, Tribology International, vol 17(6), 317-323.

2. Prabhu T J, and Ganesan N (1999). Effects of tilt on the characteristics of multirecess hydrostatic thrust bearing under conditions of no rotation, Wear, vol 92(2), 269-277.

3. Osman T A, Safar Z S et al. (2006). Experimental assessment of hydrostatic thrust bearing performance Original Research Article, Tribology International, vol 29(3), 233-239.

4. Spur G, and Patzwald R (1998). Lubrication of hydrodynamic journal bearings with magnetic fluids Production Engineering, vol 5(1), 47-50.

5. Yacout A W, Ismaeel A S et al. (2007). The combined effects of the centripetal inertia and the surface roughness on the hydrostatic thrust spherical bearing performance, Tribology International, vol 40(3), 522-532.

6. Andrés L S, and Childs D (2007). Angled injection-hydrostatic bearings, analysis and comparison to test results, Journal of Tribology, vol 119(1), 179-187.

7. Stansfield F M (1970). Hydrostatic bearings for machine tools, The Machinery Publishing Co, 1-227.

8. van Beek A, and Lepic L (2007). The combined effects of the centripetal inertia and the surface roughness on the hydrostatic thrust spherical bearing performance, Tribology International, vol 40(3), 522-532.

9. Kurtin K, Childs D et al. (1993). Experimental versus theoretical characteristics of a high-speed hybrid (combination Hydrostatic and Hydrodynamic) bearing, Journal of Tribology, vol 115(1), 160-169.

10. Andrés L S (1998). Bulk flow analysis of hybrid thrust bearings for process fluid applications, Journal of Tribology, vol 122(1), 170-180.

11. Chivens D R, and Chivens D E (1999). Impact of hydrostatic bearings on the design and performance of telescopes, SPIE Proceedings, Acquisition, Tracking, and Pointing XIII, 123, vol 3692.

12. Andrés L S (2006). A hybrid radial bearing with improved rotor dynamic stability, 1st International Conference in Rotor dynamics of Machinery, ISCORMA1, Paper 2006. 